\title{
Emphysematous Cholecystitis with Pneumobilia
}

\author{
Yee-Fung Lin Hong-Ming Chao Sheng-Chuan His \\ Division of General Surgery, Department of Surgery, Armed Forces Tao-Yuan General Hospital, Taiwan, ROC
}

A 67-year-old diabetic male presented to the emergency department with severe right hypochondrium pain of 3 days' duration, accompanied with fever and vomiting. The temperature was $38.2^{\circ} \mathrm{C}$, and Murphy's sign was detected. Plain abdominal X-ray film revealed a markedly circular gas pattern (fig. 1) in the right upper quadrant. Subsequent computed tomography (CT) confirmed the presence of air in the gallbladder wall (fig. 2)

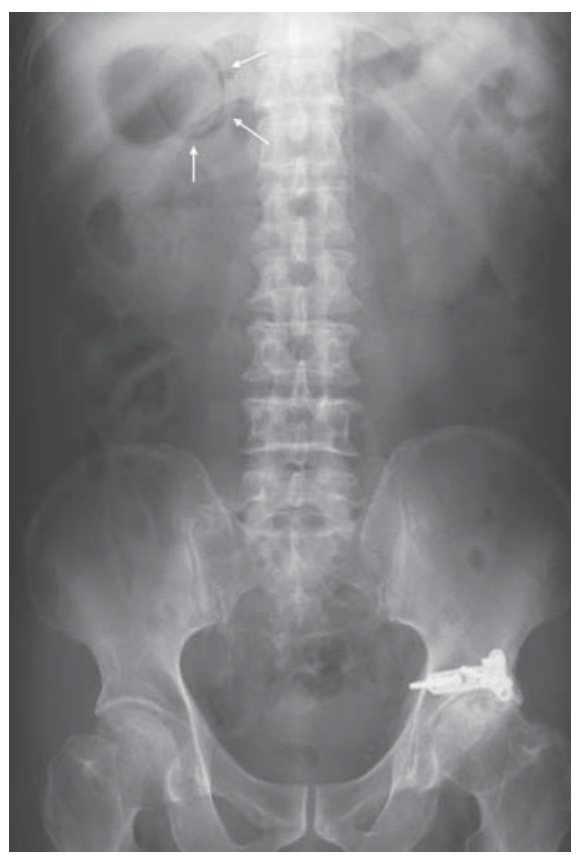

Fig. 1. Plain-film radiography showing gas around the gallbladder (arrows).

\section{KARGER}

Fax +4161306 1234 E-Mail karger@karger.ch www.karger.com (c) 2009 S. Karger AG, Basel

0253-4886/09/0261-0020\$26.00/0

Accessible online at:

www.karger.com/dsu and an air-fluid level. The CT also showed the air extending into the common bile duct and the biliary tree (fig. 3). Emergency open cholecystectomy was performed. The pathology report confirmed acute necrotizing cholecystitis and Clostridium perfringens was isolated from the bile culture. The patient was treated postoperatively with broad-spectrum antibiotic therapy and recovered uneventfully.

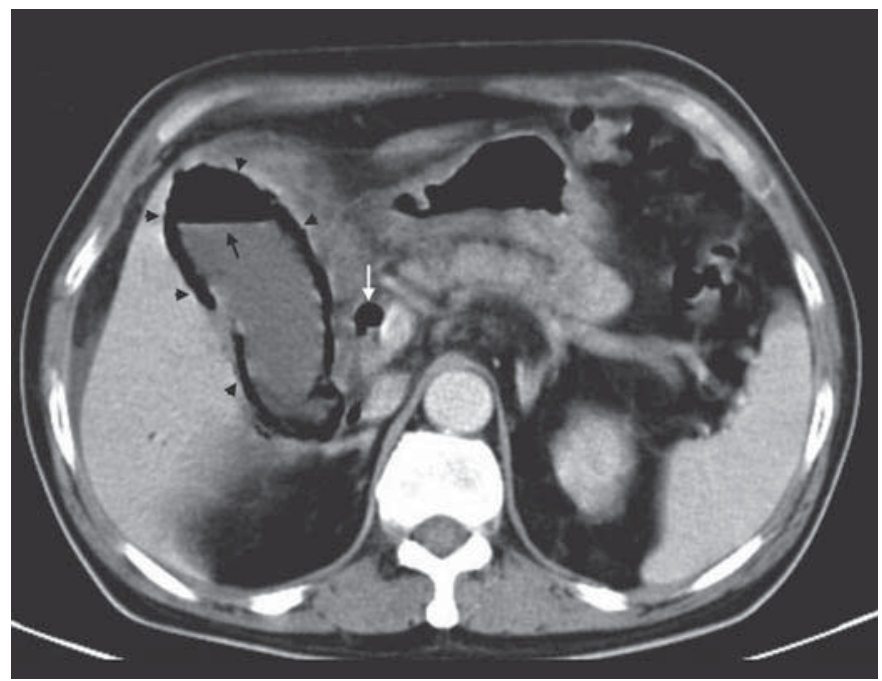

Fig. 2. CT image showing air within the gallbladder wall (arrowheads) and air-fluid level in the gallbladder (black arrow) that extends into the common bile duct (white arrow). 


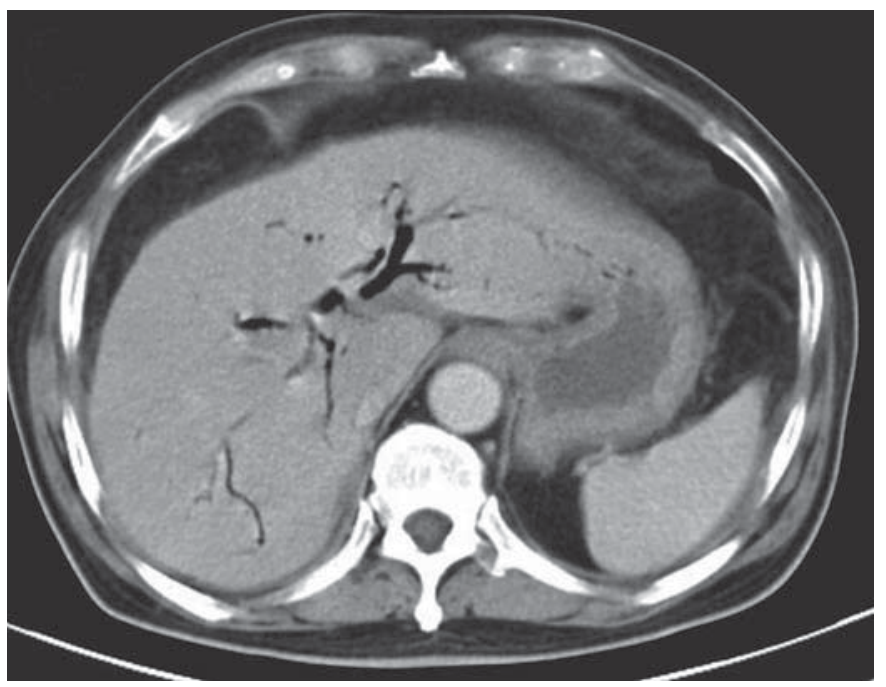

Fig. 3. CT image showing air in the biliary tree.
Emphysematous cholecystitis is a separate pathological entity to acute cholecystitis. It has a tendency to progress to gangrene and perforation of the gallbladder, and the overall mortality is substantially higher than in patients with acute cholecystitis (15\% vs. less than $4 \%$ ) [1]. Definitive treatment is cholecystectomy, and temporary cholecystostomy is also recommended to control sepsis for patients in poor condition [2]. Hence, prompt diagnosis and early surgical intervention are undoubtedly needed to minimize the serious morbidity and mortality rates associated with emphysematous cholecystitis.
References
- 1 Mentzer RM, Golden GT, Chandler JG, Horsley JS: A comparative appraisal of emphysematous cholecystitis. Am J Surg 1975; 129:10-15.

-2 Slot WB, Ooms HW, Van der Werf SD, Puylaert JB: Percutaneous gallbladder drainage in emphysematous cholecystitis. Neth J Med 1995;46:86-89. 